Pacific Journal of Mathematics

TWO APPLICATIONS OF THE UNIT NORMAL BUNDLE OF A 


\title{
TWO APPLICATIONS OF THE UNIT NORMAL BUNDLE OF A MINIMAL SURFACE IN $R^{N}$
}

\author{
NORIO EJIRI \\ Dedicated to Professor Shingo Murakami on his sixtieth birthday
}

A Gauss parametrization of a minimal surface in $R^{3}$ is well known. We prove a generalization.

THEOREM A. Let $U$ be an open set of $S^{N}(1)$ and $f$ a function on $U$ such that

$$
\Delta_{S} N_{(1)} f=-N f
$$

and 0 is an eigenvalue of Hess $f+f\langle$,$\rangle of multiplicity N-2$, where $\langle$,$\rangle is the metric of S^{N}(1)$ and $\Delta_{S} N_{(1)}$ is the Laplacian of $S^{N}(1)$. Then the map of $U$ into $R^{N+1}$ defined by

$$
f \eta+\operatorname{grad} f
$$

is of rank 2 and gives a minimal surface, where $\eta$ is the identity map on $S^{N}(1)$. Conversely, for a minimal surface $M$ in $R^{N+1}$, a neighborhood of each point of $M$ without geodesic points has this representation.

If $M$ is a complete orientable minimal surface of finite total curvature, then there is a global representation $(*)$ of $M$. Using this idea, we obtain the following.

THEOREM B. Let $M$ be a complete orientable minimal surface of finite total curvature in $R^{N+1}$. Then there exist a positive real number $c(N)$ depending on $N$ such that

$$
\operatorname{index}(M) \leq c(N) \int(-K) * 1_{M},
$$

where $K$ is the Gauss curvature of $M$ and $* 1_{M}$ is the area form of $M$.

Theorem B gives an answer for an open question posed by Cheng and Tysk in [CY1]. After this paper was submitted, the author learned that Cheng and Tysk in [CT2] obtained a similar result as Theorem B by using another Gauss map (generalized Gauss map). 
Finally we consider a generalization of minimal herissons [RT].

I'd like to thank Professors J. Eells, J. Rawnsley, M. Micallef and $\mathrm{K}$. Ohshika for their hospitality while visiting at the University of Warwick.

2. Second variation formula. Let $M$ be a minimal surface in $R^{N+1}$ and $\chi$ the immersion. Let $U(M)$ be the unit normal bundle of the normal bundle $N(M)$. Then we define a Gauss map $G$ of $U(M)$ into the $N$-dimensional unit sphere $S^{N}(1)$ by $G(x, \eta)=\eta$ for $(x, \eta) \in$ $U(M)$. $G$ induces a degenerate Riemannian metric of constant curvature 1 on $U(M)$. Let $\xi$ be a section of $N(M)$ with compact support. Then a function $F_{\xi}$ on $U(M)$ is defined by

$$
F_{\xi}(x, \eta)=\langle\xi, \eta\rangle,
$$

where $(x, \eta) \in U(M)$. Let $I(\xi, \xi)$ be the second variation of the area functional in the direction of $\xi$. Then we get

Proposition 2.1.

$$
I(\xi, \xi)=((N-1) / \omega) \int\left(\left|\nabla F_{\xi}\right|^{2}-N F_{\xi}^{2}\right) * 1_{U(M)},
$$

where $\omega$ is the volume of $S^{N-2}(1)$ and $* 1_{U(M)}$ is the volume form of $U(M)$.

This is well known in the case of $N=2$.

Proof. Let $x$ be a point of $M$ and $e_{\alpha}$ for $\alpha=3, \ldots, N+1$ be a local orthonormal framing of $N(M)$ such that

$$
\nabla_{X}^{\perp} e_{\alpha}=0 \text { for all tangent vectors } X \text { at } x,
$$

where $\nabla^{\perp}$ is the normal connection of $N(M)$. Furthermore we may consider that the second fundamental form $A_{\eta}$ in the direction of $\eta$ is diagonal and given by

$$
\left(\begin{array}{cc}
\lambda & 0 \\
0 & -\lambda
\end{array}\right)
$$

Then we get $G_{*}\left(\tilde{e}_{1}\right)=-\lambda e_{1}, G_{*}\left(\tilde{e}_{2}\right)=\lambda e_{2}$ and $G_{*}(\zeta)=\zeta$, where $\tilde{e}_{1}, \tilde{e}_{2}$ are horizontal lifts of principal vectors $e_{1}, e_{2}$ at $x$ to the tangent space of $U(M)$ at $(x, \eta)$ and $\zeta$ is a normal vector with $\langle\eta, \zeta\rangle=0$. Thus the induced metric is given by

$$
\left(\begin{array}{llll}
\lambda^{2} & & & \\
& \lambda^{2} & & \\
& & 1 & \\
& & & 1
\end{array}\right)
$$


and the volume form is $\lambda^{2} * 1_{M} * 1_{S^{N}(1)}$. Note that $\lambda^{2}$ is $(1 / 2)\left|A_{\eta}\right|^{2}$. Since

$$
\tilde{e}_{1} F_{\xi}=\left\langle\nabla_{e_{i}}^{\perp} \xi, \eta\right\rangle \text { and } \zeta F_{\xi}=\langle\xi, \zeta\rangle
$$

we have

$$
\left|\nabla F_{\xi}\right|^{2}=\left((1 / \lambda)^{2} \sum\left\langle\nabla_{e_{l}}^{\perp} \xi, \eta\right\rangle^{2}\right)+|\xi|^{2}-F_{\xi}^{2},
$$

which implies

$$
\int\left|\nabla F_{\xi}\right|^{2} * 1_{U(M)}=\int(1 / 2)\left|\nabla F_{\xi}\right|^{2}\left|A_{\eta}\right|^{2} * 1_{M} * 1_{S^{N-2}(1)} .
$$

Now we have the integral over the fibre at $x$ as follows:

$$
\begin{aligned}
& \int(1 / 2)\left|\nabla F_{\xi}^{2}\right|\left|A_{\eta}^{2}\right| * 1_{S^{N-2}(1)} \\
& =\int\left\{\sum\left\langle\nabla_{e_{\imath}}^{\perp} \xi, \eta\right\rangle^{2}+(1 / 2)\left|A_{\eta}\right|^{2}|\xi|^{2}-(1 / 2)\left|A_{\eta}\right|^{2} F_{\xi}^{2}\right\} * 1_{S^{N-2}(1)} .
\end{aligned}
$$

When we put $\eta=\sum y^{\alpha} e_{\alpha}$, we have

$$
\begin{aligned}
\int & \left\{\sum\left\langle\nabla_{e_{\imath}}^{\perp} \xi, \eta\right\rangle^{2}\right\} * 1_{S^{N-2}(1)} \\
= & \int\left\{\sum y^{\alpha} y^{\beta}\left\langle\nabla_{e_{\imath}}^{\perp} \xi, e_{\alpha}\right\rangle\left\langle\nabla_{e_{\imath}}^{\perp} \xi, e_{\beta}\right\rangle\right\} * 1_{S^{N-2}(1)} .
\end{aligned}
$$

It follows from

$$
\int y^{\alpha} y^{\beta} * 1_{S^{N-2}(1)}=(\omega /(N-1)) \delta_{\alpha \beta}
$$

that we obtain

$$
\int\left\{\sum\left\langle\nabla_{e_{l}}^{\perp} \xi, \eta\right\rangle^{2}\right\} * 1_{S^{N-2}(1)}=(\omega /(N-1))\left|\nabla^{\perp} \xi\right|^{2}
$$

and

$$
\begin{aligned}
& \int(1 / 2)\left|A_{\eta}\right|^{2}|\xi|^{2} * 1_{S^{N-2}(1)} \\
& =(1 / 2) \int\left\{\sum h_{i j}^{\alpha} h_{i j}^{\beta} y^{\alpha} y^{\beta}|\xi|^{2}\right\} * 1_{S^{N-2}(1)} \\
& =(\omega / 2(N-1))|\sigma|^{2}|\xi|^{2},
\end{aligned}
$$

where $h_{i j}^{\alpha}=\left\langle A_{e_{\alpha}} e_{i}, e_{j}\right\rangle$ and $|\sigma|^{2}=\sum h_{i j}^{\alpha} h_{i j}^{\alpha}$. On the other hand, since

$$
\begin{aligned}
& \int(1 / 2)\left|A_{\eta}\right|^{2} F_{\xi}^{2} * 1_{S^{N-2}(1)} \\
& =(1 / 2) \int\left\{\sum h_{i j}^{\alpha} h_{i j}^{\beta} y^{\alpha} y^{\beta} y^{\gamma} y^{\delta}\left\langle e_{\gamma}, \xi\right\rangle\left\langle e_{\delta}, \xi\right\rangle\right\} * 1_{S^{N-2}(1)}
\end{aligned}
$$


holds and we may consider $e_{3}=\xi /|\xi|$, by

$$
\int y^{\alpha} y^{\beta}\left(y^{3}\right)^{2} * 1_{S^{N-2}(1)}=(\omega /(N+1)(N-1))\left(\delta_{\alpha \beta}+2 \delta_{3 \alpha} \delta_{3 \beta}\right),
$$

we obtain

$$
\begin{aligned}
& (1 / 2) \int\left|A_{\eta}\right|^{2} F_{\xi}^{2} * 1_{S^{N-2}(1)} \\
& =(\omega / 2(N+1)(N-1))|\sigma|^{2}|\xi|^{2} \\
& \quad+(\omega(N+1)(N-1))\left\{\sum\left\langle\xi, \sigma_{i j}\right\rangle\left\langle\xi, \sigma_{i j}\right\rangle\right\},
\end{aligned}
$$

where $\sigma_{i j}=\sum h_{i j}^{\alpha} e_{\alpha}$. Thus we have

$$
\begin{aligned}
& \int\left(\left|\nabla F_{\xi}\right|^{2}-N F_{\xi}^{2}\right) * 1_{U(M)} \\
& \quad=(\omega /(N-1)) \int\left(\left|\nabla^{\perp} \xi\right|^{2}-\sum\left\langle\xi, \sigma_{i j}\right\rangle\left\langle\xi, \sigma_{i j}\right\rangle\right) * 1_{M} .
\end{aligned}
$$

PROPOSITION 2.2. Let $\xi$ be a normal vector field of $N(M)$. Then $\xi$ is a Jacobi field if and only if

$$
\Delta_{U(M)} F_{\xi}=-N F_{\xi}
$$

Proof. We fix a point $(x, \eta)$ of $U(M)$. Let $\gamma(s)$ be a geodesic with arc length parameter $s$ such that $\gamma(0)=x$. We denote by $X$ the tangent of $\gamma(s)$ at $x$. Let $e_{1}$ and $e_{2}$ be the principal vectors of $A_{\eta}$ such that $A_{\eta} e_{1}=\lambda e_{1}$ and $A_{\eta} e_{2}=-\lambda e_{2}$ and $e_{1}(s)$ and $e_{2}(s)$ the parallel vector fields along $\gamma(s)$ with respect to the connection of $T(M)$ such that $e_{1}(0)=e_{1}$ and $e_{2}(0)=e_{2}$. Let $e_{\alpha}, \alpha=3, \ldots, e_{N+1}$ be an orthonormal basis of $N_{x}(M)$ and $e_{\alpha}(s)$ the parallel vector fields along $\gamma(s)$ with respect to $\nabla^{\perp}$ such that $e_{\alpha}(0)=e_{\alpha}$. We may set $e_{3}(0)=\eta$. Then $\left(\gamma(s), e_{3}(s)\right)$ is the horizontal lift of $\gamma(s)$ through $(x, \eta)$ in $U(M)$. By the definition of $G$, we obtain

$$
G_{*}\left(\tilde{\gamma}_{*}(s)\right)=-A_{e_{3}(s)} \gamma_{*}(s)
$$

Let $\widetilde{\nabla}$ be the covariant differentiation with respect to the degenerate metric induced by $G$. Then we have

$$
\begin{aligned}
G_{*}\left(\widetilde{\nabla}_{\tilde{\gamma}_{*}(0)} \tilde{\gamma}_{*}(s)\right)= & \text { the component of }\left[-d A_{e_{3}(s)} \gamma_{*}(s) / d s\right]_{s=0} \\
& \text { orthogonal to } \eta .
\end{aligned}
$$


It follows that

$$
\begin{aligned}
\widetilde{\nabla}_{\tilde{\gamma}_{*}(0) \tilde{\gamma}_{*}(s)=} & \left(\left\langle\eta,\left(\nabla_{X} \sigma\right)\left(X, e_{1}\right)\right\rangle / \lambda\right) \tilde{e}_{1} \\
& -\left(\left\langle\eta,\left(\nabla_{X} \sigma\right)\left(X, e_{2}\right)\right\rangle / \lambda\right) \tilde{e}_{2} \\
& -\sum_{\alpha=4}^{N-1} \sum_{k=1}^{2}\left\langle\eta, \sigma\left(X, e_{K}\right)\right\rangle\left\langle e_{\alpha}, \sigma\left(X, e_{K}\right)\right\rangle_{\alpha} .
\end{aligned}
$$

It is easy to extend $e_{\alpha}$ for $\alpha=4, \ldots, N-1$ to the vertical vector fields $\tilde{e}_{\alpha}$ on $U(M)$ such that

$$
\widetilde{\nabla}_{\tilde{e}_{\alpha x}} \tilde{e}_{\alpha}=0 \text { at }(x, \eta) \text {. }
$$

Furthermore, for the horizontal lift $\tilde{Y}$ of a vector field $Y$ defined on a neighborhood at $x$, we have

$$
\widetilde{\nabla}_{\tilde{e}_{n}} \tilde{Y}=\left(\left\langle A_{e_{r}} Y, e_{1}\right\rangle / \lambda\right) \tilde{e}_{1}-\left(\left\langle A_{e_{r}} Y, e_{2}\right\rangle / \lambda\right) \tilde{e}_{2} \quad \text { at }(x, \eta) .
$$

Using these vector fields, we obtain the following for each point $(x, \eta)$ $\in U(M)$.

$$
\begin{aligned}
\operatorname{Hess} F_{\xi}(X, X)= & \left\langle\eta, \operatorname{Hess} \xi(X, X)+\sum\left\langle\sigma\left(X, e_{k}\right), \xi\right\rangle \sigma\left(X, e_{k}\right)\right\rangle \\
& -\left\langle\eta,\left(\nabla_{X} \sigma\right)\left(X, e_{1}\right)\right\rangle\left\langle\eta \cdot \nabla_{e_{1}}^{\perp} \xi\right\rangle / \lambda \\
& +\left\langle\eta,\left(\nabla_{X} \sigma\right)\left(X, e_{2}\right)\right\rangle\left\langle\eta \cdot \nabla_{e_{2}}^{\perp} \xi\right\rangle / \lambda \\
& -\sum\left\langle\eta, \sigma\left(X, e_{k}\right)\right\rangle^{2} F_{\xi},
\end{aligned}
$$

Hess $F_{\xi}\left(e_{\alpha}, e_{\alpha}\right)=-F_{\xi}$ for $\alpha=4, \ldots, N-1$

Hess $F_{\xi}\left(X, e_{\alpha}\right)=\left\langle e_{\alpha}, \nabla_{X}^{\perp} \xi-\left(\left\langle\eta, \nabla_{e_{1}}^{\perp} \xi\right\rangle / \lambda\right) \sigma\left(X, e_{1}\right)\right.$$$
\left.+\left(\left\langle\eta, \nabla_{e_{2}}^{\perp} \xi\right\rangle / \lambda\right) \sigma\left(X, e_{2}\right)\right\rangle \text {. }
$$

Thus we have

$$
\Delta_{U(M)} F_{\xi}=-(1 / \lambda)^{2}\langle\eta, J(\xi)\rangle-N F_{\xi},
$$

where $J$ is the Jacobi operator of $N(M)$.

We know that $\chi^{\perp}=\sum\left\langle\chi, e_{\alpha}\right\rangle e_{\alpha}$ is a Jacobi field, where $\chi$ is the position vector of $M$. By the calculation as in Proposition 2.2, we obtain

Lemma 2.1. Hess $F_{\chi}^{\perp}+F_{\chi}^{\perp}\langle$,$\rangle has an eigenvalue 0$ of multiplicity $N-2$ at $(x, \eta) \in U\left(\stackrel{M}{)}\right.$ such that $\operatorname{det} A_{\eta} \neq 0$. 
Now we may consider that $F_{\chi}^{\perp}$ is locally a function on an open set $U$ of $S^{N}(1)$. Then we define a map of $U$ into $R^{N+1}$ such that

$$
F_{\chi}^{\perp \eta}+\operatorname{grad} F_{\chi}^{\perp}
$$

By a simple calculation, it is just $\chi$. Conversely let $f$ be an eigenfunction of eigenvalue $N$ on an open set $U$ in $S^{N}(1)$ such that the eigenvalue of the Hess $f+f \backslash$,$\rangle has 0$ of multiplicity $N-2$. Then

$$
f \eta+\operatorname{grad} f
$$

is a map of rank 2 and hence gives a minimal surface. Thus we obtain a Gauss parametrization of a minimal surface in $R^{N+1}$.

As a generalization of Theorem A, we easily obtain the following.

Proposition 2.3. Let $U$ be an open set of $S^{N}(1)$ and $f$ a function on $U$ such that Hess $f+f\langle$,$\rangle has an eigenvalue 0$ of multiplicity $N-m$. Then $f \eta+\operatorname{grad} f$ is a map of $U$ into $R^{N+1}$ of rank $m$ and furthermore gives an m-dimensional submanifold such that the $(m-1) s t$ mean curvature vector vanishes. We call the representation the Gauss parametrization by an eigenfunction. Conversely let $M$ be an $m$-dimensional submanifold in $R^{N+1}$ such that the $(m-1)$ st mean curvature vector vanishes, then a neighborhood of each point such that $\operatorname{det} A_{\eta} \neq 0$ for some normal vector $\eta$ the Gauss parametrization by an eigenfunction.

REMARK. In [DG], similar constructions are presented.

COROllary 2.1. Let $M$ be a complex m-dimensional Kaehler submanifold in $C^{N+1}$. Then a neighborhood of each point such that $\operatorname{det} A_{\eta} \neq 0$ for some normal vector $\eta$ admits the Gauss parametrization by an eigenfunction.

Proof. It is well known that the $(2 m-1)$ st mean curvature vector vanishes on $M$.

Let $M$ be a minimal surface in $R^{N+1}$ and $\xi$ a Jacobi field. Then Proposition 2.2 implies that $F_{\xi}$ is an eigenfunction of eigenvalue $N$. We define the rank $\gamma_{\xi}$ of Jacobi field by $N-\mu$, where $\mu$ is the multiplicity of eigenvalue 0 of

$$
\text { Hess } F_{\xi}+F_{\xi}\langle,\rangle \text {. }
$$

By Proposition 2.3, we have a $\gamma_{\xi}$-dimensional submanifold with zero $\left(\gamma_{\xi}-1\right)$ st mean curvature vector. For example, let $M$ be a minimal 
surface in $R^{3}$. Then $\gamma_{\xi}=0$ or 2 holds for a Jacobi field $\xi$ and if $\gamma_{\xi}=2$ holds, then we obtain a minimal surface

$$
\xi-\sum\left(A_{\eta}^{-1}\right)^{i j}\left\langle\nabla_{e_{i}}^{\perp} \xi, \eta\right\rangle e_{j},
$$

which gives a minimal deformation of $M-$ \{geodesic points $\}$ whose normal variation vector field is $\xi$. In fact

$$
\chi+s\left\{\xi \sum\left(A_{\eta}^{-1}\right)^{i j}\left\langle\nabla_{e_{i}}^{\perp} \xi, \eta\right\rangle e_{j}\right\}
$$

is a one parameter family of minimal surfaces, where $\chi$ is the immersion of $M$ into $R^{3}$.

Next let $M$ be a minimal surface in $R^{4}$ and $\xi$ a Jacobi field. Then $\gamma_{\xi}$ is 0,2 or 3 . In the case of $\gamma_{\xi}=3$, we have a hypersurface of zero second mean curvature in $R^{4}$, which implies zero scalar curvature. Thus the first given minimal surface is a limit of deformation of hypersurfaces of zero scalar curvature in $R^{4}$.

3. The index of minimal surfaces. Let $M$ be a complete orientable minimal surface of finite total curvature in $R^{N+1}$. Then there exists a compact orientable Riemann surface $\bar{M}$ and finite points $p_{1}, \ldots, p_{q}$ $\in \bar{M}$ such that $M$ is conformally equivalent to $\bar{M}-\left\{p_{1}, \ldots, p_{q}\right\}$ and the generalized Gauss map of $M$ into $G_{2}\left(R^{N+1}\right)$ is extendable over $\bar{M}$. Let $L$ be the tautological vector bundle over $G_{2}\left(R^{N+1}\right)$ with rank $N-1$. Then the restriction of the induced bundle over $\bar{M}$ to $M$ is the normal bundle $N(M)$. So the unit sphere bundle $U(\bar{M})$ over $\bar{M}$ gives a compactification of $U(M)$ such that the ends are fibres at $p_{i}$. It is clear that the map $G$ is extendable on $U(\bar{M})$ and we denote by $\bar{G}$ the map. Note that $\bar{G}$ is real analytic.

LEMMA 3.1. The degenerate set $S$ for $\bar{G}$ is an analytic set of codimension $\geq 2$ if $M$ is not in some $R^{3}$.

Proof. It is clear that $S$ is an analytic set. Assume that $S$ has an open set of $U(M)$. Then as analytic function $\left|A_{\eta}\right|^{2}$ on $U(M)$ is zero on some open set, which implies that $M$ is plane. Assume that $S$ has codimension 1. Then we note that the rank of $\left.\theta\right|_{S}$ is 1 or 2 , where $\theta$ is the projection of $U(M)$ onto $M$. If the rank is 2 , there is an open set $U$ of $M$ such that each fibre at $x \in U$ has an $(N-3)$ dimensional submanifold where $\left|A_{\eta}^{2}\right| \equiv 0$. For each $x \in U$, we have an orthonormal basis $e_{3}, \ldots, e_{N+1}$ such that, for all $\alpha \geq 5$,

$$
A_{e_{3}}=\left(\begin{array}{cc}
\lambda & 0 \\
0 & -\lambda
\end{array}\right), \quad A_{e_{4}}=\left(\begin{array}{cc}
0 & \mu \\
\mu & 0
\end{array}\right), \quad A_{e_{\alpha}}=\left(\begin{array}{cc}
0 & 0 \\
0 & 0
\end{array}\right)
$$


and hence, for any unit normal vector $\eta=a e_{3}+b e_{4}+$ others, $\operatorname{det} A_{\eta}=$ 0 holds if and only if $a^{2} \lambda^{2}+b^{2} \mu^{2}=0$, which implies that if $\lambda \neq 0$ and $\mu \neq 0$, then the set where $\operatorname{det} A_{\eta}=0$ is an $(N-4)$-dimensional sphere. It is a contradiction and hence $\lambda$ or $\mu=0$, which implies the first normal space on $U$ is at most 1-dimensional. It is easy to see that $M$ is in some $R^{3}$. Next assume that the rank of $\left.\theta\right|_{S}$ is 1 . Then, on the image $\theta(S)$, the second fundamental form of $M$ vanishes. On the other hand, it is well known that totally geodesic points are isolated. It is contradiction.

By the result in $[\mathbf{H}]$, we can have a stratification of $S$ such that if a stratum $T$ satisfies $T \cap S \neq \varnothing$, then $S \supset T$. So $\bar{G}(S)$ has a stratification and $\bar{G}^{-1}(\bar{G}(S))$ is a sum of finite stratums of codimension $\geq 2$. By a simple argument, we get

LEMMA 3.2 .

$$
\bar{G}: U(\bar{M}) \backslash \bar{G}^{-1}(\bar{G}(S)) \rightarrow S^{N}(1) \backslash \bar{G}(S)
$$

is a $k$-sheeted covering map, where $k$ is the total curvature of $M / 2 \pi$.

From Proposition 2.1, we obtain the following:

$$
\begin{aligned}
\text { index }(M) \leq & \text { the number of eigenvalues of } \Delta_{U(\bar{M})} \text { that are } \\
& \text { strictly less than } N .
\end{aligned}
$$

Let $\left\{\lambda_{i}\right\}_{i=0}^{\infty}$ and $\left\{\mu_{i}\right\}_{i=0}^{\infty}$ be eigenvalues of $\Delta_{S} N_{(1)}$ and $\Delta_{U(\bar{M})}$, respectively. A theorem in [5], together with Lemma 3.2 implies

$$
\sum e^{-\mu_{\imath} t} \leq k\left(\sum e^{-\lambda_{\imath} t}\right) \text {. }
$$

Thus we conclude that

$$
(\operatorname{index}(M)) e^{-N t} \leq \sum_{\mu_{i}<N} e^{-\mu_{t} t} \leq \sum e^{-\mu_{t} t} \leq k\left(\sum e^{\lambda_{t} t}\right) .
$$

Hence

$$
\text { index }(M) \leq e^{N t}\left(\sum e^{-\lambda_{i} t}\right) k .
$$

Note that if $M$ is not in some $R^{3}$, then $c(N)$ is given by

$$
2 \pi \inf _{t>0}\left\{e^{N t}\left(\sum e^{-\lambda_{t} t}\right)\right\}
$$

4. A generalization of minimal herissons. Recently Rosenberg and Toubiana [RT] give some results on complete minimal finite branched 
surfaces in $R^{3}$ of finite total curvature $4 \pi$, which are called minimal herissons and parametrized by their Gauss image.

Let $M$ be an $m$-dimensional submanifold of zero $(m-1)$ st mean curvature vector in $R^{N+1}$. We consider the following condition $(* *)$.

(**) There exist finite stratum $S$ of $U(M)$ and $S^{\prime}$ of $S^{N}(1)$

such that codimensions of elements of $S$ and $S^{\prime} \geq 2$

and

$G: U(M) \backslash S \rightarrow S^{N}(1) \backslash S^{\prime}$

is a $k$-sheeted covering.

Let $\mathfrak{M}$ denote the space of $m(2 \leq m \leq N)$-dimensional submanifolds of zero $(m-1)$ st mean curvature vector in $R^{N+1}$ which satisfy $(* *)$. Following as in $[\mathbf{R T}]$, we can define a sum operation in $\mathfrak{M}$ :

$$
\begin{aligned}
& M_{1}+M_{2}=\left\{\sum \theta\left(x_{i}\right)+\sum \theta\left(y_{l}\right): G_{1}^{-1}(z)=\left\{x_{i}\right\},\right. \\
& \left.G_{2}^{-1}(z)=\left\{y_{i}\right\}, \text { where } z \in S^{N}(1) \backslash S_{1}^{\prime} \cup S_{2}^{\prime}\right\},
\end{aligned}
$$

where $G_{1}$ and $G_{2}$ are the Gauss map of $M_{1}$ and $M_{2}$, respectively and $S_{1}^{\prime}$ and $S_{2}^{\prime}$ satisfy $(* *)$ for $G_{1}$ and $G_{2}$. Note that the equality of dimensions of $M_{1}$ and $M_{2}$ is not necessary. This operation may be considered as follows: for $z \in S^{N}(1) \backslash S_{1}^{\prime} \cup S_{2}^{\prime}$, we define a function $f$ by

$$
f(z)=\sum F_{\chi_{1}}^{\perp}\left(x_{l}\right)+\sum F_{\chi_{2}}^{\perp}\left(y_{i}\right),
$$

where $\chi_{1}$ and $\chi_{2}$ are immersions of $M_{1}$ and $M_{2}$ into $R^{N+1}$, respectively. It is clear that

$$
\Delta_{S} N_{(1)} f=-N f
$$

on $U=S^{N}(1) \backslash S_{1}^{\prime} \cup S_{2}^{\prime}$ and hence $f$ is analytic on $U$. By the analyticity of $f$ on $U$, the multiplicity of the eigenvalue 0 of Hess $f+f\langle$, is constant $N-m$ on some open dense set of $U$. Thus we get an $m$-dimensional submanifold of zero $(m-1)$ st mean curvature vector in $R^{N+1}$ which gives $M_{1}+M_{2}$.

Proposition 4.1. Assume that $M_{1}+M_{2}$ is of dimension $m$. Then $M_{1}+M_{2}$ is of zero $(m-1)$ st mean curvature vector and parametrized by Gauss image. In particular, the total absolute curvature is the volume of $S^{N}(1)$.

REMARK. The study of $f$ which satisfies $\Delta_{S} N_{(1)} f=-N f$ has a relation to $N$-dimensional space-like minimal submanifolds of constant curvature 1 in an $(N+2)$-dimensional deSitter space time [K]. 
In [N], Nayatani proves that, if $M$ be a complete orientable minimal surface of finite total curvature, then $M$ has a finite index. But it does not imply the existence of $c(N)$.

\section{REFERENCES}

[CT1] S. Y. Cheng and J. Tysk, An index characterization of the catenoid and index bounds for minimal surfaces in $\mathbf{R}^{4}$, Pacific J. Math., 134 (1988), 251-260.

[CT2] __ Schrodinger operators and index bounds for minimal submanifolds, preprint.

[DG] M. Dajczer and D. Gromoll, Gauss parametrizations and rigidity aspects of submanifolds, J. Differential Geom., 22 (1985), 1-12.

[K] M. Kotani, The minimal submanifolds in a pseudo space form and Gauss inverse images in the hyperbolic space, preprint.

[N] S. Nayatani, On the Morse index of complete minimal surfaces in Euclidean space, preprint.

[RT] H. Rosenberg and E. Toubiana, Complete minimal surfaces and minimal herissons, J. Differential Geom., 28 (1988), 115-132.

[T] J. Tysk, Eigenvalue estimates with applications to minimal surfaces, Pacific J. Math., 128 (1987), 361-366.

Received January 6, 1989, the author was partially supported by a grant of the Japanese Education Ministry.

College of General Education

NAGOYA UNIVERSITY

ChiKusa-Ku, Nagoya 464, JaPAN 


\title{
PACIFIC JOURNAL OF MATHEMATICS EDITORS
}

\author{
V. S. VARADARAJAN \\ (Managing Editor) \\ University of California \\ Los Angeles, CA 90024-1555-05 \\ Herbert Clemens \\ University of Utah \\ Salt Lake City, UT 84112 \\ THOMAS ENRIGHT \\ University of California, San Diego \\ La Jolla, CA 92093
}

R. FINN

Stanford University

Stanford, CA 94305

Hermann FlaschKa

University of Arizona

Tucson, AZ 85721

VAUGHaN F. R. JoNes

University of California

Berkeley, CA 94720

Steven Kerckhoff

Stanford University

Stanford, CA 94305

\section{C. MOORE}

University of California

Berkeley, CA 94720

Martin ScharlemanN

University of California

Santa Barbara, CA 93106

HAROLD STARK

University of California, San Diego

La Jolla, CA 92093
R. ARENS
E. F. BECKENBACH
F. WolF
K. YoshidA

\section{ASSOCIATE EDITORS}

\begin{tabular}{ll}
\multicolumn{1}{c}{ SUD-1982) } & \multicolumn{1}{c}{$(1904-1989)$} \\
UNIVERSITY OF ARIZONA & UNIVERSITY OF OREGON \\
UNIVERSITY OF BRITISH COLUMBIA & UNIVERSITY OF SOUTHERN CALIFORNIA \\
CALIFORNIA INSTITUTE OF TECHNOLOGY & STANFORD UNIVERSITY \\
UNIVERSITY OF CALIFORNIA & UNIVERSITY OF HAWAII \\
MONTANA STATE UNIVERSITY & UNIVERSITY OF TOKYO \\
UNIVERSITY OF NEVADA, RENO & UNIVERSITY OF UTAH \\
NEW MEXICO STATE UNIVERSITY & WASHINGTON STATE UNIVERSITY \\
OREGON STATE UNIVERSITY & UNIVERSITY OF WASHINGTON \\
\hline
\end{tabular}

The Supporting Institutions listed above contribute to the cost of publication of this Journal, but they are not owners or publishers and have no responsibility for its content or policies.

\begin{abstract}
Mathematical papers intended for publication in the Pacific Journal of Mathematics should be in typed form or offset-reproduced (not dittoed), double spaced with large margins. Please do not use built up fractions in the text of the manuscript. However, you may use them in the displayed equations. Underline Greek letters in red, German in green, and script in blue. The first paragraph must be capable of being used separately as a synopsis of the entire paper. In particular it should contain no bibliographic references. Please propose a heading for the odd numbered pages of less than 35 characters. Manuscripts, in triplicate, may be sent to any one of the editors. Please classify according to the 1980 Mathematics Subject Classification (1985 Revision) scheme which can be found in the December index volumes of Mathematical Reviews. Supply name and address of author to whom proofs should be sent. All other communications should be addressed to the managing editor, or Elaine Barth, University of California, Los Angeles, California 90024-1555-05.

There are page-charges associated with articles appearing in the Pacific Journal of Mathematics. These charges are expected to be paid by the author's University, Government Agency or Company. If the author or authors do not have access to such Institutional support these charges are waived. Single authors will receive 50 free reprints; joint authors will receive a total of 100 free reprints. Additional copies may be obtained at cost in multiples of 50 .
\end{abstract}

The Pacific Journal of Mathematics (ISSN 0030-8730) is published monthly except for July and August. Regular subscription rate: $\$ 190.00$ a year (10 issues). Special rate: $\$ 95.00$ a year to individual members of supporting institutions.

Subscriptions, orders for numbers issued in the last three calendar years, and changes of address should be sent to Pacific Journal of Mathematics, P.O. Box 969, Carmel Valley, CA 93924, U.S.A. Old back numbers obtainable from Kraus Periodicals Co., Route 100, Millwood, NY 10546.

The Pacific Journal of Mathematics at P.O. Box 969, Carmel Valley, CA 93924 (ISSN 0030-8730) is published monthly except for July and August. Second-class postage paid at Carmel Valley, California 93924, and additional mailing offices. Postmaster: send address changes to Pacific Journal of Mathematics, P.O. Box 969, Carmel Valley, CA 93924.

PUBLISHED BY PACIFIC JOURNAL OF MATHEMATICS, A NON-PROFIT CORPORATION Copyright (C) 1991 by Pacific Journal of Mathematics 